\title{
Dual-Frequency Decoupling Networks for Compact Antenna Arrays
}

\author{
Jacob Carl Coetzee \\ School of Engineering Systems, Queensland University of Technology, P.O. Box 2434, Brisbane, QLD 4001, Australia \\ Correspondence should be addressed to Jacob Carl Coetzee, jacob.coetzee@qut.edu.au
}

Received 25 November 2010; Accepted 12 January 2011

Academic Editor: Mattia Borgarino

Copyright () 2011 Jacob Carl Coetzee. This is an open access article distributed under the Creative Commons Attribution License, which permits unrestricted use, distribution, and reproduction in any medium, provided the original work is properly cited.

Decoupling networks can alleviate the effects of mutual coupling in antenna arrays. Conventional decoupling networks can provide decoupled and matched ports at a single frequency. This paper describes dual-frequency decoupling which is achieved by using a network of series or parallel resonant circuits instead of single reactive elements.

\section{Introduction}

The adverse effects of mutual coupling on the performance of multiport antennas are well known [1]. The effects can be countered by using a decoupling network which provides an additional signal path to effectively cancel the external coupling between array elements to yield decoupled ports [2-4].

In its simplest form, the decoupling network consists of reactive elements connected between neighbouring array elements, but this approach only applies when the mutual admittances between elements are all purely imaginary [2]. The design of decoupling networks for arrays with arbitrary complex mutual admittances has been described [3-5]. In [6], closed-form design equations for the decoupling network elements of symmetrical 2-element and 3-element arrays were presented. This concept was extended to the decoupling of larger, circulant symmetric arrays through repeated decoupling of the eigenmodes [7]. However, these methods are only applicable to the decoupling of arrays over a small bandwidth at a single frequency. This paper describes dual-frequency decoupling of arrays. The procedure is based on the methods described in $[6,7]$, but where each reactive element in the single-frequency decoupling network is replaced with either a series or parallel combination of an inductor and a capacitor in order to achieve simultaneous decoupling and matching at two frequencies.

\section{Theory}

The procedures described in [6] or [7] may be employed to design a network at two distinct frequencies, $f_{1}$ and $f_{2}$. Subsequently, the relations provided in [8] can be used to design $L$-section impedance matching networks which match the decoupled port impedances to the system impedance $Z_{0}$ at $f_{1}$ and $f_{2}$. Refer, for example, to the decoupling and matching networks for a 2-element array, shown in Figure $1 . X_{11}$ and $B_{21}$ are the elements of the decoupling network at frequency $f_{1}$, while $X_{12}$ and $B_{22}$ are the corresponding values at frequency $f_{2}$. The element values are obtained in closed form from [6]. Ports $1^{\prime}$ and $2^{\prime}$ will thus be decoupled at both frequencies, but the port impedances $Z_{1}^{\prime}=R_{1}^{\prime}+j X_{1}^{\prime}$ and $Z_{2}^{\prime}=R_{2}^{\prime}+j X_{2}^{\prime}$ are not matched to $Z_{0}$. The matching networks shown in Figure 1 are for the case where $R_{1}^{\prime}>Z_{0}$ and $R_{2}^{\prime}>Z_{0} . B_{31}$ and $X_{41}$ and are the matching network elements at frequency $f_{1}$, whereas $B_{32}$ and $X_{42}$ are the elements at frequency $f_{2}$.

In order to realise the required values of the elements of the decoupling and matching networks, each reactive element in Figure 1 can be replaced with a circuit consisting of either a series or a parallel combination of a capacitor and an inductor. The component values of the capacitors and inductors can be computed using the relations provided in Table 1. The chosen topology in Figure 2 sees the series and parallel elements in Figure 1 replaced by series and parallel $L-C$ circuits, respectively. Note that the design equations 
TABLE 1: Design equations for calculation of the parallel or series $L-C$ circuit implementations of reactive elements in the decoupling and matching networks. Here, $\omega_{j}=2 \pi f_{j}$ represents the angular frequency.

\begin{tabular}{|c|c|c|}
\hline Network elements & Parallel/series & Component values \\
\hline \multirow{4}{*}{$X_{i 1}, X_{i 2}$} & & $C_{i}=\left(\omega_{1} / X_{i 1}-\omega_{2} / X_{i 2}\right) /\left(\omega_{2}^{2}-\omega_{1}^{2}\right)$ \\
\hline & Parallel & $L_{i}=\left(\omega_{1} / X_{i 1}+\omega_{1}^{2} C_{i}\right)^{-1}$ \\
\hline & Series & $L_{i}=\left(\omega_{2} X_{i 2}-\omega_{1} X_{i 1}\right) /\left(\omega_{2}^{2}-\omega_{1}^{2}\right)$ \\
\hline & & $C_{i}=\left(\omega_{1}^{2} L_{i}-\omega_{1} X_{i 1}\right)^{-1}$ \\
\hline \multirow{4}{*}{$B_{i 1}, B_{i 2}$} & Paralle & $C_{i}=\left(\omega_{2} B_{i 2}-\omega_{1} B_{i 1}\right) /\left(\omega_{2}^{2}-\omega_{1}^{2}\right)$ \\
\hline & & $L_{i}=\left(\omega_{1}^{2} C_{i}-\omega_{1} B_{i 1}\right)^{-1}$ \\
\hline & Series & $L_{i}=\left(\omega_{1} / B_{i 1}-\omega_{2} / B_{i 2}\right) /\left(\omega_{2}^{2}-\omega_{1}^{2}\right)$ \\
\hline & Neitis & $C_{i}=\left(\omega_{1}^{2} L_{i}+\omega_{1} / B_{i 1}\right)^{-1}$ \\
\hline
\end{tabular}

TABLE 2: Decoupling and matching network elements and component values of the circuit in Figure 2.

\begin{tabular}{lccc}
\hline & Array scattering parameters & Decoupling network elements & Matching network elements \\
\hline \multirow{2}{*}{ Frequency $=f_{1}$} & $S_{11}^{a}=-9.27 \mathrm{~dB} \angle 137.6^{\circ}$ & $X_{11}=-29.7052 \Omega$ & $B_{31}=-0.0132 \Omega^{-1}$ \\
& $S_{12}^{a}=-4.95 \mathrm{~dB} \angle-22.3^{\circ}$ & $B_{21}=-0.0279 \Omega^{-1}$ & $X_{41}=-47.3233 \Omega$ \\
Frequency $=f_{2}$ & $S_{11}^{a}=-7.50 \mathrm{~dB} \angle 89.5^{\circ}$ & $X_{12}=-9.9391 \Omega$ & $B_{32}=0.0101 \Omega^{-1}$ \\
& $S_{12}^{a}=-5.53 \mathrm{~dB} \angle-45.2^{\circ}$ & $B_{22}=0.0219 \Omega^{-1}$ & $X_{42}=54.5235 \Omega$ \\
\hline & & $L_{1}=7.2337 \mathrm{nH}$ & $L_{3}=0.4352 \mathrm{nH}$ \\
Implementation & $C_{1}=0.4778 \mathrm{pF}$ & $C_{3}=9.2297 \mathrm{pF}$ \\
& $L_{2}=0.2038 \mathrm{nH}$ & $L_{4}=40.638 \mathrm{nH}$ \\
\hline
\end{tabular}

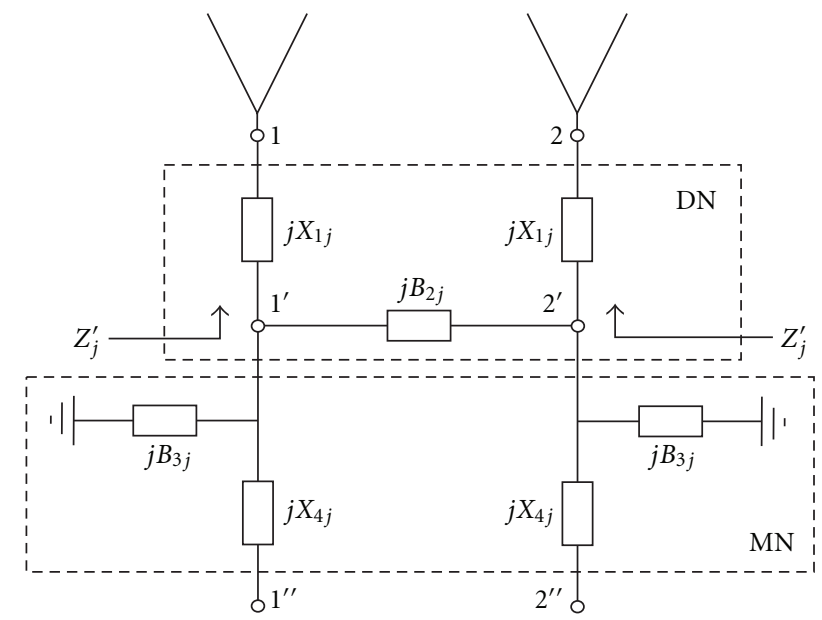

Figure 1: Decoupling network (DN) and matching network (MN) for a 2-element array at frequency $f_{j}, j=1,2$.

in [6-8] provide several possible solutions for the element values of the decoupling and matching networks in Figure 1. A number of these options would result in nonphysical, negative values for the capacitors and inductors in Figure 2. Care should therefore be taken to only select those options which yield positive values for each capacitor and inductor in the implementation.

\section{Example}

To verify the theory, dual-frequency decoupling and matching networks were designed for the same 2-element

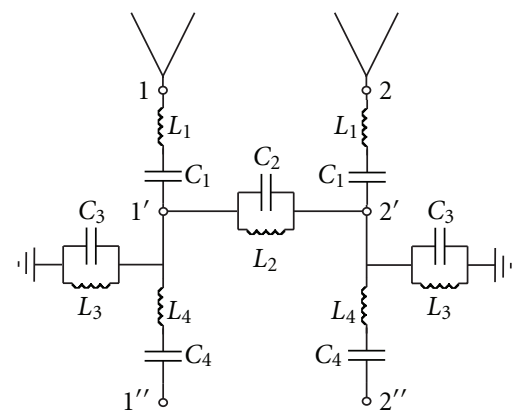

FIGURE 2: Implementation of the decoupling and matching networks for a 2-element array using parallel or series $L$ - $C$ circuits.

monopole array used in [6]. The array elements were wires measuring $\lambda / 4$ in length and $\lambda / 40$ in diameter at $2.5 \mathrm{GHz}$, and an element spacing of $\lambda / 10$ was used. A system impedance of $Z_{0}=50 \Omega$ was assumed. This array was decoupled and matched at frequencies $f_{1}=2.4 \mathrm{GHz}$ and $f_{2}=2.6 \mathrm{GHz}$. The scattering parameters, decoupling and matching network elements, and the components of the implementation are shown in Table 2. The calculated scattering parameters of the decoupled array are shown together with those of the original array in Figure 3. The results clearly illustrate the validity of the theory, since the array is both decoupled and matched at $f_{1}$ and $f_{2}$.

It should be noted that the decoupling/matching circuit is sensitive to capacitor and inductor tolerances. The decoupled 2-element monopole array was analysed repeatedly with element values varying randomly within a tolerance of 


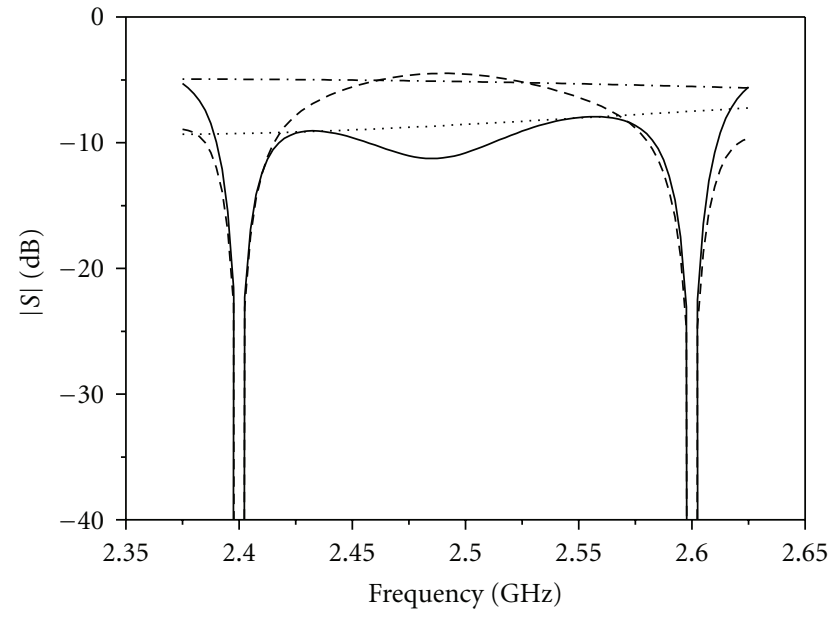

$\ldots . .\left|S_{11}\right|$ (array only) - $\left|S_{11}\right|$ (decoupled and matched) -..- $\left|S_{12}\right|$ (array only) - - $\left|S_{12}\right|$ (decoupled and matched)

Figure 3: Scattering parameters of the 2-element array, decoupled and matched at $f_{1}=2.4 \mathrm{GHz}$ and $f_{2}=2.6 \mathrm{GHz}$.

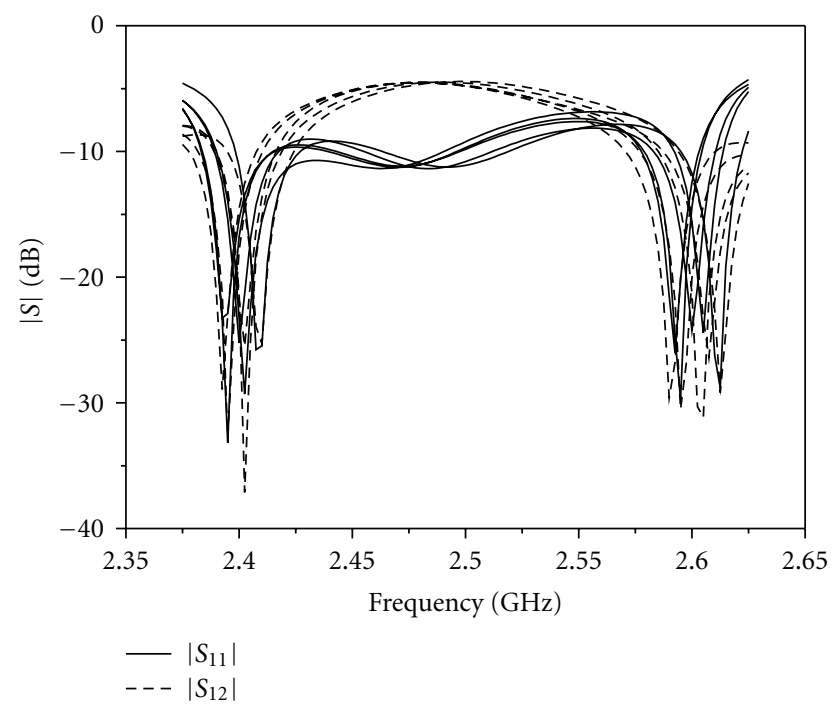

FIGURE 4: Scattering parameters of the decoupled and matched 2element array with capacitor and inductor tolerance of $\pm 1 \%$.

$\pm 1 \%$ and $\pm 5 \%$. The results are shown in Figures 4 and 5 , respectively. Although some frequency shifting is apparent in Figure 4, the performance of the system is still adequate. However, in the case of the $\pm 5 \%$ element tolerance in Figure 5, the overall performance has deteriorated beyond acceptable standards. Element tolerances will therefore be an important consideration in the practical implementation of such circuits.

\section{Conclusion}

Dual-frequency decoupling of tightly coupled arrays was described. The approach involves the design of decoupling



FIGURE 5: Scattering parameters of the decoupled and matched 2element array with capacitor and inductor tolerance of $\pm 5 \%$.

and matching networks at two distinct frequencies and implementing them simultaneously using a ladder network of parallel or series $L-C$ circuits. The approach was illustrated for a 2-element array, but is equally applicable to larger arrays. Single-frequency decoupling networks are usually characterised by narrow bandwidths. By selecting the two frequencies of a dual-frequency decoupling network close to the desired operating frequency, the bandwidth for a singlefrequency application can possibly also be improved.

\section{References}

[1] A. C. Ludwig, "Mutual coupling, gain and directivity of an array of two identical antennas," IEEE Transactions on Antennas and Propagation, vol. 24, no. 6, pp. 837-841, 1976.

[2] H. J. Chaloupka, X. Wang, and J. C. Coetzee, "A superdirective 3 -element array for adaptive beamforming," Microwave and Optical Technology Letters, vol. 36, no. 6, pp. 425-430, 2003.

[3] P. T. Chua and J. C. Coetzee, "Microstrip decoupling networks for low-order multiport arrays with reduced element spacing," Microwave and Optical Technology Letters, vol. 46, no. 6, pp. 592-597, 2005.

[4] J. Weber, C. Volmer, K. Blau, R. Stephan, and M. A. Hein, "Miniaturized antenna arrays using decoupling networks with realistic elements," IEEE Transactions on Microwave Theory and Techniques, vol. 54, no. 6, pp. 2733-2740, 2006.

[5] S. C. Chen, Y. S. Wang, and S. J. Chung, "A decoupling technique for increasing the port isolation between two strongly coupled antennas," IEEE Transactions on Antennas and Propagation, vol. 56, no. 12, pp. 3650-3658, 2008.

[6] J. C. Coetzee and Y. Yu, "Closed-form design equations for decoupling networks of small arrays," Electronics Letters, vol. 44, no. 25, pp. 1441-1442, 2008.

[7] J. C. Coetzee and Y. Yu, "Design of decoupling networks for circulant symmetric antenna arrays," IEEE Antennas and Wireless Propagation Letters, vol. 8, pp. 291-294, 2009.

[8] D. M. Pozar, Microwave Engineering, John Wiley \& Sons, Hoboken, NJ, USA, 2005. 

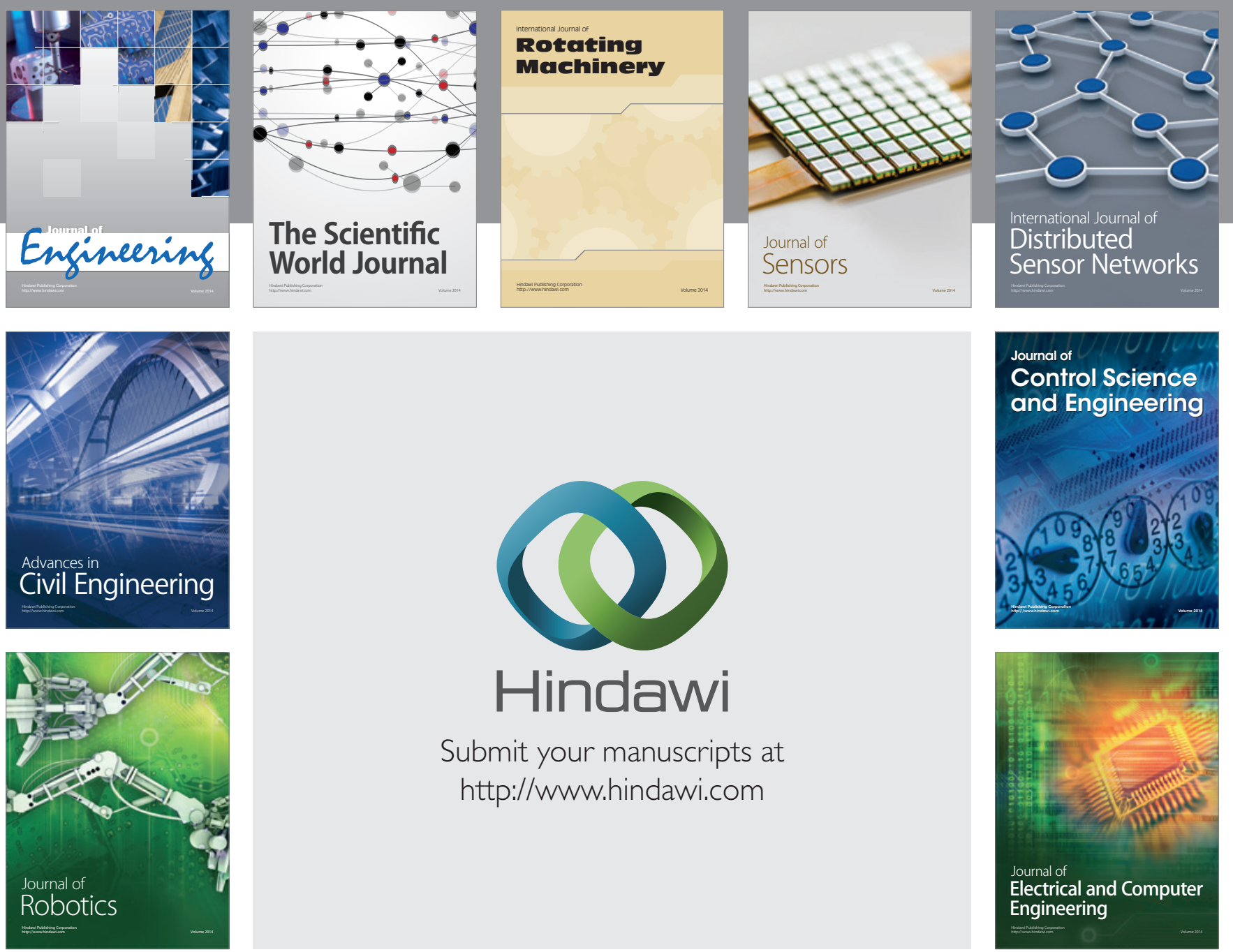

Submit your manuscripts at

http://www.hindawi.com
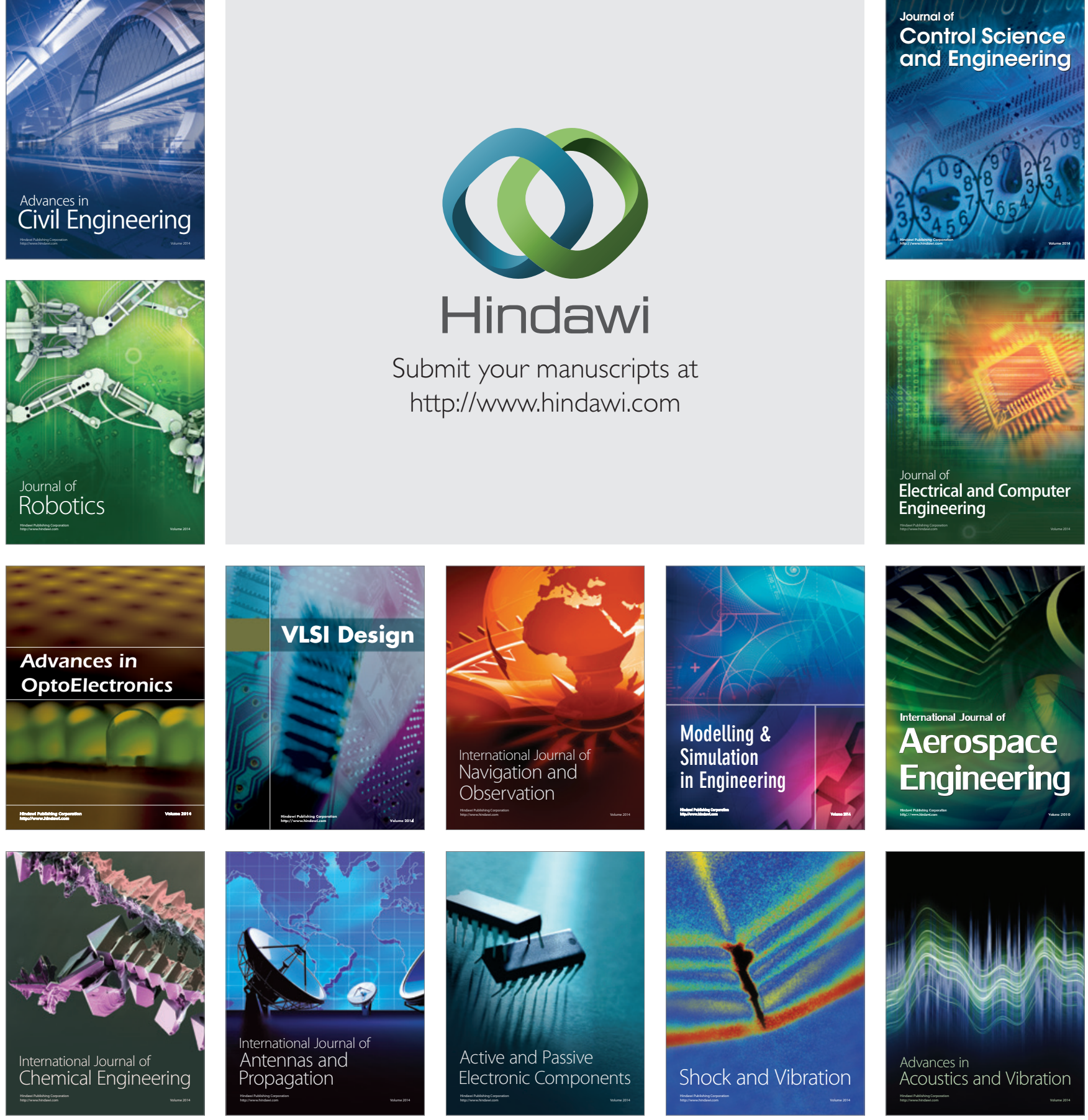